\title{
Conceptual problems in detecting the evolution of dark energy when using distance measurements
}

\author{
K. Bolejko \\ Nicolaus Copernicus Astronomical Center, Polish Academy of Sciences, Bartycka 18, 00716 Warszawa, Poland \\ e-mail: bolejko@camk.edu.pl
}

Received 16 July 2010 / Accepted 17 October 2010

\section{ABSTRACT}

\begin{abstract}
Context. Dark energy is now one of the most important and topical problems in cosmology. The first step to reveal its nature is to detect the evolution of dark energy or to prove beyond doubt that the cosmological constant is indeed constant. However, in the standard approach to cosmology, the Universe is described by the homogeneous and isotropic Friedmann models.

Aims. We aim to show that in the perturbed universe (even if perturbations vanish if averaged over sufficiently large scales) the distance-redshift relation is not the same as in the unperturbed universe. This has a serious consequence when studying the nature of dark energy and, as shown here, can impair the analysis and studies of dark energy.

Methods. The analysis is based on two methods: the linear lensing approximation and the non-linear Szekeres Swiss-Cheese model. The inhomogeneity scale is $\sim 50 \mathrm{Mpc}$, and both models have the same density fluctuations along the line of sight.

Results. The comparison between linear and non-linear methods shows that non-linear corrections are not negligible. When inhomogeneities are present the distance changes by several percent. To show how this change influences the measurements of dark energy, ten future observations with $2 \%$ uncertainties are generated. It is shown the using the standard methods (i.e. under the assumption of homogeneity) the systematics due to inhomogeneities can distort our analysis, and may lead to a conclusion that dark energy evolves when in fact it is constant (or vice versa).

Conclusions. Therefore, if future observations are analysed only within the homogeneous framework then the impact of inhomogeneities (such as voids and superclusters) can be mistaken for evolving dark energy. Since the robust distinction between the evolution and non-evolution of dark energy is the first step to understanding the nature of dark energy a proper handling of inhomogeneities is essential.
\end{abstract}

Key words. cosmological parameters - cosmology: observations - cosmology: theory - dark energy - large-scale structure of Universe

\section{Introduction}

The most important problem in contemporary cosmology is the nature of dark energy. There are many ongoing attempts to explain the nature of dark energy. The simplest explanation assumes that dark energy does not evolve and is modeled by only one single constant - the cosmological constant. In this scenario, the cosmological constant is considered as one of the fundamental constants of nature. However, most scientists are not satisfied with this type of solution and continue to investigate the nature of dark energy. Most of these attempts invoke new physics: f(R) cosmologies (Buchdahl 1970; Starobinsky 1980; Kerner 1982; Barrow \& Ottewill 1983; Barrow \& Cotsakis 1988; Li \& Barrow 2007; Koyama 2008; Moldenhauer et al. 2010), braneword models (Randall \& Sundrum 1999; Randall \& Sundrum 1999; Dvali et al. 2000; Deffayet et al. 2001; Alcaniz 2002; Sahni \& Shtanov 2003; Maia et al. 2005), scalar fields (Saini et al. 2000; Sahni \& Wang 2000; Chiba et al. 2000; Peebles \& Ratra 2003; Copeland et al. 2006), phantom fields (Caldwell et al. 2003), holographic cosmology (Kaloper \& Linde 1999; Li 2004; Hsu 2004; Simpson 2007), or accounting for exotic substances such as Chaplygin gas (Bertolami et al. 2004; Bento et al. 2003; Debnath et al. 2004). There are also alternatives which replace dark energy with an inhomogeneity of a radius of approximately one Gpc (Célérier 2000; Iguchi et al. 2002; Alnes et al. 2006; Alnes \& Amarzguioui 2006; Enqvist \& Mattsson 2007;
Bolejko 2008; García-Bellido \& Haugbølle 2008; Clifton et al. 2008; Bolejko \& Wyithe 2009; Célérier et al. 2010, for a review and explicit examples see Bolejko et al. 2009) - these, exceptionally, do not require any new physics. In most of these alternatives, an effective dark energy equation of state evolves with time. Therefore, from the observational point of view, the most important discovery would be to detect any possible changes in dark energy over the period of evolution of the Universe or to prove beyond doubt that the cosmological constant is indeed constant.

The evolution of dark energy manifests itself in its equation of state. For the perfect fluid, the conservation equations $T_{; \beta}^{\alpha \beta}=$ 0 imply Ellis (1971)

$$
\frac{\partial \rho_{\mathrm{DE}}}{\partial t}+\Theta\left(\rho_{\mathrm{DE}}+p_{\mathrm{DE}}\right)=0
$$

where $\rho$ is energy density, $p$ is pressure, and $\Theta$ is the expansion parameter (in the Friedmann models $\Theta=3 H ; H$ is the Hubble parameter). Therefore, the only case when dark energy does not evolve is when $\rho_{\mathrm{DE}}=-p_{\mathrm{DE}}$. Thus, if only $w \equiv p_{\mathrm{DE}} / \rho_{\mathrm{DE}}$ differs from -1 then dark energy evolves.

However, as long as dark energy is not directly detected any measurement of $w$ is always indirect and relies on the assumption that some relations hold. In most cases, it is assumed that the distance-redshift relation is as in the Friedmann model. This paper shows that in the perturbed universe, even if $\langle\delta \rho\rangle=0$ 
the distance relation is not the same as in the unperturbed universe. Therefore, the study of dark energy within the homogeneous framework may strongly bias the results of these analyses.

Since the distance relation in its general form is a partial differential equation, to make the reader more aware of some features of differential equations a simple example with a harmonic oscillator is presented in Sect. 2. In Sect. 3, the relation for the distance in the general inhomogeneous case is presented and an analogy with the harmonic oscillator solution is discussed. Section 4 discusses the consequences of evolving dark energy. In Sect. 5, an example of the Swiss-Cheese model is presented to shown that small-scale inhomogeneities if not analysed properly can be mistaken for an evolving dark energy.

\section{Harmonic oscillator}

We now discuss some basic features of the harmonic oscillator. Although this material is most probably well know, it is instructive to review it here, as there is a important relation to cosmology, namely to the distance relation. The evolution of the harmonic oscillator is given by

$\ddot{x}+\omega_{0}^{2} x=0$.

The solution is a combination of $\sin \omega_{0} t$ and $\cos \omega_{0} t$. We refer to this solution as the unperturbed solution. If some additional force is present (for simplicity we assume that the force is sinusoidal) then

$\ddot{x}+\omega_{0}^{2} x-F_{0} \sin \omega t=0$.

The solution of this equation is a sum of the unperturbed solution and the steady-state solution given by

$x_{s}(t)=\frac{F_{0}}{\omega_{0}-\omega} \sin \omega t$.

As seen, in this case, the driving force introduces oscillations of frequency $\omega$ around the unperturbed solution. However, if we depart from this highly idealistic scenario the solution changes. We assume that $F$ is proportional to $x\left(F=x F_{0} \sin \omega t\right)$, then

$\ddot{x}+x\left(\omega_{0}^{2}-F_{0} \sin \omega t\right)=0$.

In this case, the solution does not oscillate around the unperturbed solution. This is presented in Fig. 1, which presents the solutions of Eqs. (1)-(3). The parameters used here are $\omega_{0}=1$, $\omega=15$, and $F_{0}=5$. The unperturbed solution, Eq. (1), is given by the solid line. The dotted line presents the solution of Eq. (2) - as seen, it oscillates around the unperturbed solution. The solution of Eq. (3) is given by the dashed line: it does not oscillate around the unperturbed solutions, even though the average of $F$ over the period vanishes, i.e. $\langle F\rangle_{t}=0$.

The above conclusion is rather obvious, although we show there is a close relation between the above example and the cosmology. We demonstrate that the distance in the perturbed universe (even if $\langle\delta \rho\rangle=0$ ) does not oscillate around the homogeneous value. This has a strong implication on the analysis of cosmological observations.

\section{Distance in the inhomogeneous universe}

The Sachs equation for the angular diameter distance is Sachs (1961)

$$
\frac{\mathrm{d}^{2} D_{\mathrm{A}}}{\mathrm{d} s^{2}}=-\left(|\sigma|^{2}+\frac{1}{2} R_{\alpha \beta} k^{\alpha} k^{\beta}\right) D_{\mathrm{A}},
$$

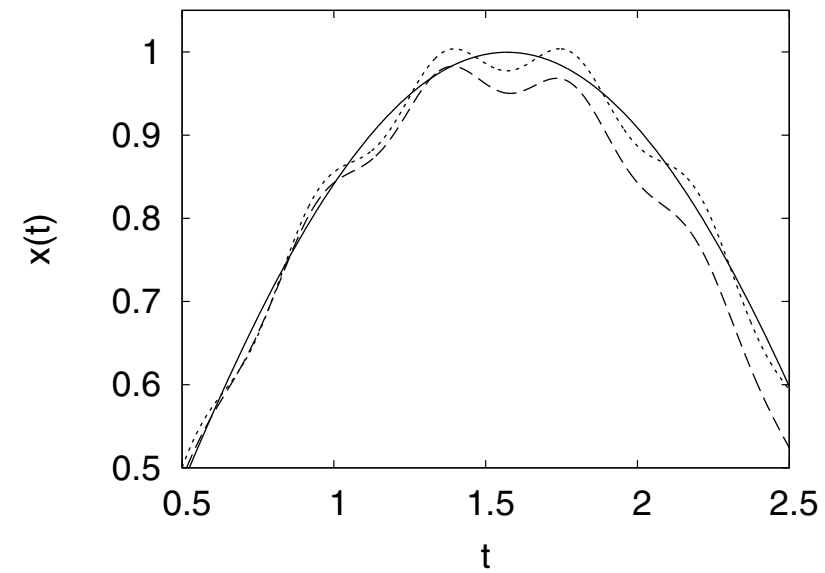

Fig. 1. The solutions of Eqs. (1)-(3). Solid line: the unperturbed solution, Eq. (1); dotted line: the solution of Eq. (2); dashed line the solution of Eq. (3).

where $D_{\mathrm{A}}$ is the angular diameter distance, $\sigma$ is the shear and vanishes in the Friedmann limit, $R_{\alpha \beta}$ is the Ricci tensor and $R_{\alpha \beta} k^{\alpha} k^{\beta}=T_{\alpha \beta} k^{\alpha} k^{\beta}$. In the comoving and synchronous coordinates, for pressure-less matter $R_{\alpha \beta} k^{\alpha} k^{\beta}=\kappa \rho k^{0} k^{0} \quad(\kappa=8 \pi$ and $G=c=1)$. Since the above relation depends linearly on $\rho$ one could naively presume that since $\langle\delta \rho\rangle=0$ then the average distance should be the same as in the unperturbed case, i.e. as in the Friedmann model.

We consider a simple example that shows otherwise: we assume that $\sigma=0$ and $R_{\alpha \beta} k^{\alpha} k^{\beta}$ is constant ${ }^{1}$. For the homogeneous model Eq. (4) has the same form as the unperturbed harmonic oscillator in Eq. (1). However, if we add density perturbations, i.e.

$\rho=\rho_{0}+\delta \rho$

then even if $\langle\delta \rho\rangle=0$ say $\delta \rho \sim \sin \omega x$, the corresponding harmonic oscillator is no longer given by Eq. (2) but by Eq. (3). Hence the solution to Eq. (4) for the perturbed universe does not oscillate about the unperturbed solution, even if $\langle\delta \rho\rangle=0$.

How does this relate to the real Universe? Is the effect large?

The real Universe on small scales is highly inhomogeneous with cosmic structures such as galaxy clusters, superclusters, and voids. Cosmic voids are of size 20-50 Mpc (Hoyle \& Vogeley 2004; Jones et al. 2009), thus to test the effect of inhomogeneities on the distance-redshift relations we consider models with similar scales of inhomogeneity. Two types of models are considered: the non-linear Swiss-Cheese model and the linear perturbative framework. The density profile along the line of sight, for both models, is presented in Fig. 2. We consider three different methods for calculating the distance:

1. Fully non-linear model:

The distance will be calculated directly from Eq. (4). The evolution of matter and null geodesics will be calculated within the Szekeres Swiss-Cheese model. The details are presented in the Appendix.

2. Lensing approximation: Within the linear perturbative scheme the distance is

$$
D_{\mathrm{A}}(z)=\bar{D}_{\mathrm{A}}\left(1+\delta_{\mathrm{D}}\right)
$$

${ }^{1}$ For the homogeneous case, we have $R_{\alpha \beta} k^{\alpha} k^{\beta} \sim a^{-5}$, so for small distance it changes relatively slowly. Even at distance around $1 \mathrm{Gpc}$, for the standard cosmological model, $a(t)=0.8 a_{0}$ (where $a_{0}$ is the scale factor at the current instant). 


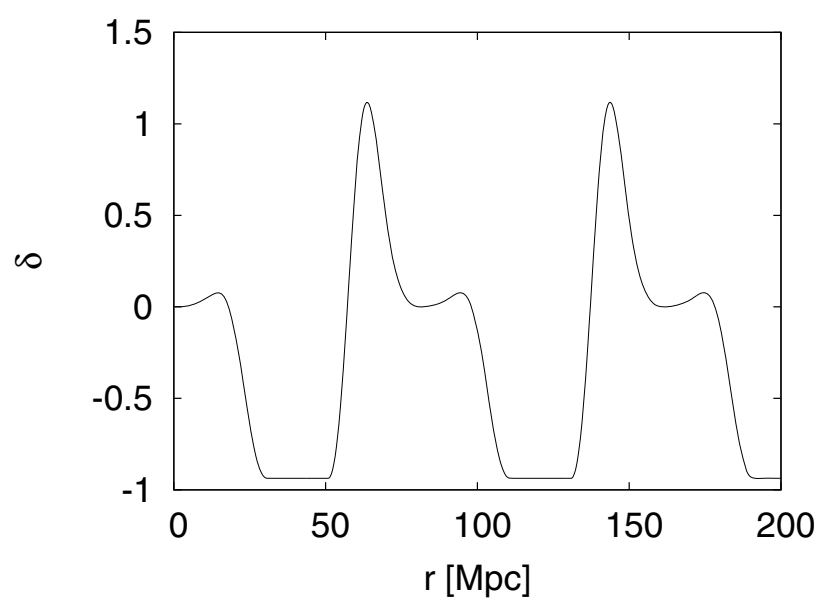

Fig. 2. Density fluctuations along the line of sight within the lensing and Swiss Cheese models.

where $\bar{D}_{\mathrm{A}}$ is the angular-diameter distance in the homogeneous universe (see Eq. (8)). The most general form for $\delta_{\mathrm{D}}$ was derived by Pyne \& Birkinshaw (2004), Bonvin et al. (2006), and Hui \& Greene (2006). Excluding the contribution from the motion of the observer and source, and taking the leading term, $\delta_{\mathrm{D}}$ reduces to

$\delta_{\mathrm{D}}=-\int_{0}^{\chi_{\mathrm{e}}} \mathrm{d} \chi \frac{\chi_{\mathrm{e}}-\chi}{\chi_{\mathrm{e}}} \chi \nabla^{2} \phi(\chi)$,

where $\chi$ is the comoving coordinate $\mathrm{d} \chi=\mathrm{d} z / H(z)$ and $\phi$ is the gravitational potential that relates to the density perturbations $\delta \rho$ via the Poisson equation $\nabla^{2} \phi=\frac{4 \pi G}{c^{2}} a^{2} \delta \rho$. Equation (6) is equivalent to the convergence in the lensing approximation and is known as the Born approximation.

The present-day density fluctuations along the line of sight are exactly the same as in the Szekeres Swiss-Cheese model mentioned above. This allows us to see how large higherorder corrections are. The evolution of $\delta$ is calculated using the linear approximations (Peebles 1980)

$\ddot{\delta}+2 \frac{\dot{a}}{a} \delta=\frac{4 \pi G}{c^{2}} \delta \rho$.

3. Standard, homogeneous, Friedmann model:

The distance within the homogeneous Friedmann model is

$\bar{D}_{\mathrm{A}}(z)=\frac{c}{H_{0}(1+z) \sqrt{-\Omega_{k}}} \sin \left(\sqrt{-\Omega_{k}} \int_{0}^{z} \mathrm{~d} z^{\prime} \frac{H_{0}}{H\left(z^{\prime}\right)}\right)$,

and

$$
\begin{aligned}
H^{2}= & H_{0}^{2}\left\{\Omega_{m}(1+z)^{3}+\Omega_{k}(1+z)^{2}+\Omega_{\mathrm{DE}}\right. \\
& \left.\times \exp \left[3 \int_{0}^{z} \frac{1+w\left(z^{\prime}\right)}{1+z^{\prime}} \mathrm{d} z^{\prime}\right]\right\},
\end{aligned}
$$

where $w(z)$ is the dark energy equation of state, and is parametrized as

$w(z)=w_{0}+w_{1} \frac{z}{1+z}$.

\section{Evolution of dark energy}

If dark energy is some kind of dynamical field, then its energy density evolves. Unfortunately, evolving dark energy cannot be modelled using the Szekeres model. The Szekeres model is the solution of the Einstein equations with a dust source + the cosmological constant, and hence cannot be used to model systems with evolving pressure. However, evolving dark energy can be modelled using the lensing approximation, but only in the case when dark energy is homogeneous. This is because Eq. (5) assumes that the expansion rate, which enters via $\bar{D}_{\mathrm{A}}$, behaves as in the homogeneous model.

When dark energy is a dynamical fluid, then two cases need to be considered: a clustering dark energy and non-clustering dark energy. If dark energy behaves as a relativistic fluid (like radiation) then its perturbations are described only by decaying modes (Mukhanov 2005). In this case, dark energy should remain almost homogeneous.

However, if dark energy clusters (like matter) then its presetday distribution can be as inhomogeneous as matter distribution. Then the lensing approximation (i.e. Eq. (6)) cannot be applied. This is because when dark energy is inhomogeneous then the average of the expansion rate will not be the same as the expansion rate of the background model, i.e. $\langle H\rangle \neq H_{0}$. This is a consequence of gradients in the pressure. If dark energy is inhomogeneous and pressure gradients are non-negligible then the $g_{00}$ component of the metric must be position dependent. We denote this by $\alpha$, i.e. $g_{00}=\alpha^{2}(t, r)$ and for simplicity consider the spherical case. Then (Bolejko \& Lasky 2008)

$\frac{\alpha^{\prime}}{\alpha}=-\frac{p^{\prime}}{\epsilon+p}$,

where $\epsilon=\rho_{\mathrm{de}}+\rho_{\text {mat }}, \rho_{\mathrm{de}}$ is the energy density of dark energy, and $\rho_{\text {mat }}$ is the energy density of matter. To estimate $\alpha$ we assume $w_{\mathrm{de}} \approx-1$ which implies that $\epsilon+p \approx \rho_{\text {mat }}$ and $-p^{\prime} \approx \rho_{\mathrm{de}}^{\prime}$. We also assume that the distribution of dark energy closely follows the matter distribution, and that $\rho_{\mathrm{de}} \approx 3 \rho_{\text {mat }}$ which imply that

$\alpha \approx \alpha_{o}\left(\frac{\rho}{\rho_{o}}\right)^{3} \approx 1+3 \delta$,

where the subscript mat has been suppressed and the subscript "o" denotes the "observer's position", i.e. $\alpha_{o}=\alpha(r=0) \equiv$ $1, \rho_{o}=\rho_{\text {mat }}(r=0)$. The above approximation shows that inhomogeneities in dark energy will have a non-negligible effect on the dynamics of the Universe. Also, since $\alpha \neq 1$ the result of averaging is different than when $\alpha=1$ (i.e. when the pressure gradients are negligible). For example, Eq. (A.13) becomes

$\langle H\rangle \approx \frac{4 \pi}{V} \int_{0}^{r} \mathrm{~d} r\left(R^{2} \dot{R}\right)^{\prime} \alpha \approx \frac{4 \pi}{V} \int_{0}^{r} \mathrm{~d} r\left(R^{2} \dot{R}\right)^{\prime}(1+3 \delta)$.

Since $\delta$ is non-zero, $\alpha$ deviates from unity and therefore

$\langle H\rangle \neq \frac{\dot{R}}{R}=H_{0}$.

This feature was also observed within the second-order perturbative scheme. Clarkson et al. (2009) showed that the average quantities (such as the expansion rate) within the second-order perturbative framework are not the same as in the unperturbed background, in other words $\langle H\rangle \neq H_{0}$. Thus, if dark energy can cluster and is as inhomogeneous as the present-day matter distribution, then not only the distance fails to oscillate about the unperturbed value but also the expansion rate. Therefore, in the next section when studying evolving dark energy it is implicitly assumed that dark energy is homogeneous. 

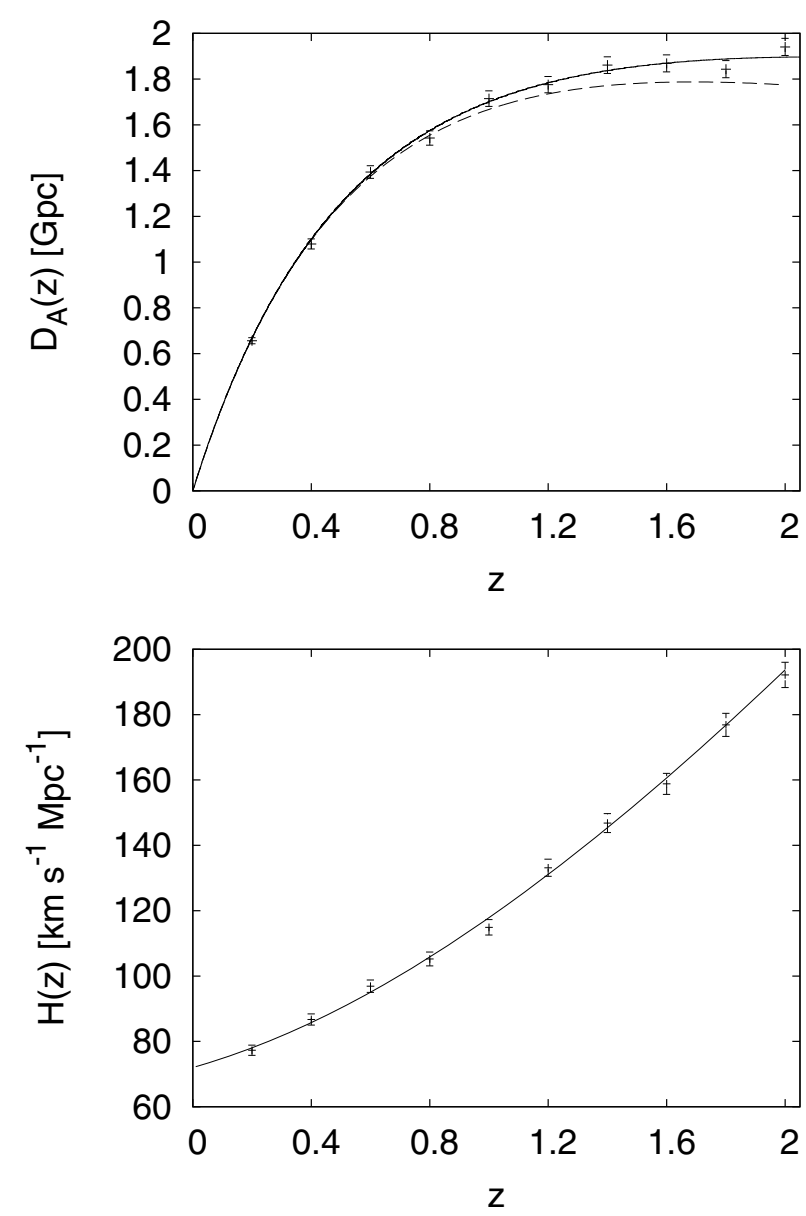

Fig. 3. The Szekeres Swiss-Cheese model (solid line), the $\Lambda \mathrm{CDM}$ model (dashed line), and future observations with $2 \%$ precision scattered around the Swiss-Cheese model. Upper panel presents $D_{\mathrm{A}}(z)$ and lower panel presents the $H(z)$ in the Swiss-Cheese and $\Lambda$ CDM model. Since the average $H(z)$ in the Swiss-Cheese model is almost the same as in the background model (see Eq. (A.13)), there is no visible difference between these two models.

\section{Future observations and dark energy}

To test the effects of inhomogeneities on the results of the analysis of cosmological observations, we consider the following example. We assume that we have data from future, very precise measurements of $D_{\mathrm{A}}$ and $H$ - say ten measurements of the baryon acoustic oscillations (BAO) at redshifts $0.2,0.4,0.6, \ldots, 1.8,2$, each measurement having $2 \%$ errors. We then take the Szekeres model and the lensing approximation, and in each case generate mock observations (ten BAO data points). An example of these data is presented in Fig. 3, where generated observations are scattered around the Szekeres model. We later analyse these mock observations using the standard methods, i.e. to find the equation of state of dark energy ( $w_{0}$ and $w_{1}$ ) we analyse the observations by fitting Eqs. (8) and (9) to the data. Two scenarios are considered: non-evolving dark energy (i.e. the cosmological constant) and an evolving one.

The results are presented in Fig. 4. The case of non-evolving dark energy is presented in the left and middle panels. As seen if the data is analysed only using the distance measurements, the inferred equation of state of dark energy differs from $w=-1$. This is because the inhomogeneities are not taken into account in the distance-redshift Eq. (8). On the other hand, the analysis of the $H(z)$ data agrees with the true equation of state of dark energy. This is because, within the Swiss-Cheese model and within the lensing approximation, the average expansion rate coincides with the unperturbed value. However, as pointed out in Sect. 4, when dark energy is not homogeneous, pressure gradients imply that $\langle H\rangle \neq H_{0}$. In this case, if pressure gradients are large and not taken into account then the inferred value from $H(z)$ data may also differ from the true one.

The evolving dark energy case is presented in the right panel of Fig. 4. Only the lensing approximation is considered here, as the Szekeres model cannot be used to describe the evolution of dark energy. As seen, the results are very similar to the case of non-evolving dark energy.

The above examples show the importance of inhomogeneities, as not taking them into account can lead to large systematics, particularly when measuring the dark energy equation of state. Evolution of dark energy is a subtle effect, hence even small deviations of $D_{\mathrm{A}}(z)$ from the standard case might introduce significant changes in the interpretation of cosmological observations. Another important result is that non-linear effects are important. As seen by comparing the left and middle panels of Fig. 4, the non-linear corrections cannot be ignored.

\section{Conclusions}

How do inhomogeneities that are present and observed in the Universe influence the distance-redshift relation?

This question has been frequently addressed since the papers by Kristian \& Sachs (1966) and Dyer \& Roeder (1972) (see also Räsänen 1999, and references therein). However, it is still common among cosmologists to disregard the effect of inhomogeneities. The common reasoning is based on the following two premises: 1) even if density variations are large the fluctuations of the gravitational potential are small and therefore the perturbation scheme can be applied; and 2) since perturbations are Gaussian they vanish after averaging and therefore they should have little impact on observations.

However, as shown in this paper the distance-redshift relation does depend on density fluctuations (not on gravitational potential), and secondly, even if perturbations vanish after averaging they do modify the distance-redshift relation and the final result deviates from the homogeneous one. Even within the perturbative scheme, it is apparent that inhomogeneities do affect the distance-redshift relation (Bonvin et al. 2006; Vanderveld et al. 2007; Gurzadyan \& Kocharyan 2009). Since the evolution of dark energy is a subtle effect, inhomogeneities cannot be ignored.

The main aim of this paper was therefore to show the importance of inhomogeneous models in the studies of dark energy. Ignoring inhomogeneities can have serious consequences since even small-scale inhomogeneities do affect the observations, especially when it comes to the equation of state of dark energy. This paper uses the explicit example to show that if cosmological observations are analysed within the framework of homogeneous models then matter inhomogeneities might be mistaken for evolving dark energy. This is a consequence of Eq. (4), which is a differential equation for the angular diameter distance. As in the case of non-idealistic harmonic oscillator, the perturbed solution does not oscillate around the unperturbed value. As trivial as it sounds, this statement must be emphasized because a large number of cosmologists seems to believe otherwise.

The results of this paper imply that systematics due to inhomogeneities can distort our analysis, and may lead to a conclusion that dark energy evolves when in fact it is constant (or vice 

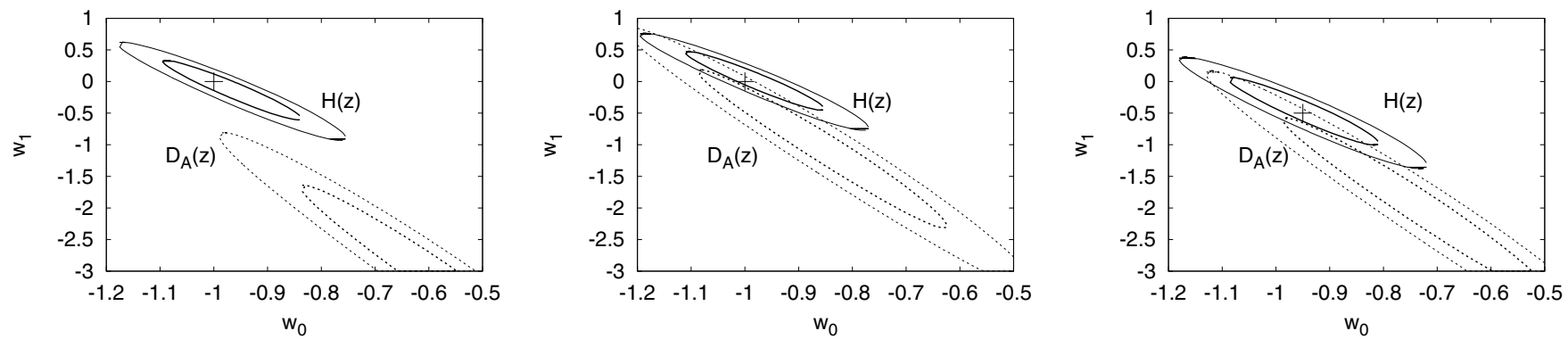

Fig. 4. 68\% and 95\% CL for the parameters $w_{0}$ and $w_{1}$ inferred from future (mock) observations. Solid lines present the constraints from $H(z)$ data, dotted line from $D_{\mathrm{A}}(z)$ data, the cross marks the true value of $w_{0}$ and $w_{1}$ used to generate the data. The misalignment of distance-constraints is a result of not taking into account inhomogeneities. Left: the results of analysis within the non-linear Swiss-Cheese model $\left(w_{0}=-1\right.$ and $\left.w_{1}=0\right)$. Middle: the results of analysis within the linear approximation scheme $\left(w_{0}=-1\right.$ and $\left.w_{1}=0\right)$. Right: the results of analysis within the linear approximation scheme $\left(w_{0}=-0.95\right.$ and $\left.w_{1}=-0.5\right)$.

versa). Since the robust distinction between the evolution and non-evolution of dark energy is the first step to understanding its nature, a proper handling of systematics is essential. Without taking into account all systematics, the precision cosmology will not be an accurate cosmology (Peebles 2010).

\section{Appendix A: Szekeres Swiss-Cheese model}

The metric of the Szekeres model has the form

$\mathrm{d} s^{2}=\mathrm{d} t^{2}-\frac{\left(R^{\prime}-R E^{\prime} / E\right)^{2}}{1-k} \mathrm{~d} r^{2}-\frac{R^{2}}{E^{2}}\left(\mathrm{~d} x^{2}+\mathrm{d} y^{2}\right)$,

where

$E=\frac{S}{2}\left[\left(\frac{x-P}{S}\right)^{2}+\left(\frac{y-Q}{S}\right)^{2}+1\right]$,

and the prime denotes the partial derivative with respect to $r$, $R^{\prime}=\partial R / \partial r$. The Einstein equations for the dust are

$\dot{R}^{2}=\frac{2 M}{R}-k+\frac{1}{3} \Lambda R^{2}$,

$\kappa \rho=\frac{2 M^{\prime}-6 M E^{\prime} / E}{R^{2}\left(R^{\prime}-R E^{\prime} / E\right)}$

where the dot denotes the partial derivative with respect to $t$, $\dot{R}=\partial R / \partial t, \Lambda$ is the cosmological constant, and $M$ is an arbitrary function of $r$. The shear is

$\sigma_{\beta}^{\alpha}=\frac{1}{3}\left(\frac{\dot{R}^{\prime}-\dot{R} R^{\prime} / R}{R^{\prime}-R E^{\prime} / E}\right) \operatorname{diag}(0,2,-1,-1)$.

The bang time function follows from (A.3)

$$
\int_{0}^{\Phi} \frac{\mathrm{d} \widetilde{\Phi}}{\sqrt{-k+2 M / \widetilde{\Phi}+\frac{1}{3} \Lambda \widetilde{\Phi}^{2}}}=t-t_{B}(r)
$$

The Weyl curvature decomposed into its electric and magnetic part is

$$
\begin{aligned}
& E_{\beta}^{\alpha}=\frac{M\left(3 R^{\prime}-R M^{\prime} / M\right)}{3 R^{3}\left(R^{\prime}-R E^{\prime} / E\right)} \operatorname{diag}(0,2,-1,-1), \\
& H^{\alpha}{ }_{\beta}=0 .
\end{aligned}
$$

The construction of the Szekeres Swiss-Cheese model closely follows Bolejko \& Célérier (2010) and the reader is referred there for more details. The functions that define the Szekeres model were chosen to be of the form

$M=M_{0}+ \begin{cases}M_{1} \ell^{3} & \text { for } \ell \leqslant x_{a}, \\ M_{2} \exp \left[-\left(\frac{\ell-2 x_{a}}{x_{a}}\right)^{2}\right] & \text { for } x_{a} \leqslant \ell \leqslant 3 x_{a} \\ -M_{1}\left(\ell-4 x_{a}\right)^{3} & \text { for } 3 x_{a} \leqslant \ell \leqslant 4 x_{a}, \\ 0 & \text { for } \ell \geqslant 4 x_{a},\end{cases}$

where $\ell=r / \mathrm{kpc}, M_{0}=\left(4 \pi G / 3 c^{2}\right) \rho_{b} \ell^{3}, \rho_{b}=\Omega_{m} \frac{3 H_{0}^{2}}{8 \pi G}, \Omega_{m}=$ $0.25, H_{0}=72 \mathrm{~km} \mathrm{~s}^{-1} \mathrm{Mpc}^{-1}, x_{a}=10^{4}, M_{1}=x_{a}^{-3} M_{2} \mathrm{e}^{-1.5}$, $M_{2}=-7 \times 10^{11} \mathrm{kpc}$.

The bang time function is $t_{B}=0$, which means that the age of the universe is the same everywhere. The function $k$ then follows from Eq. (A.6). And the functions $S, P$, and $Q$ are

$S=\left(5 \times 10^{3}+\ell\right)^{ \pm 0.78}$

$P=1=x_{0}$,

$Q=1=y_{0}$,

where + is for propagation from the origin $\left[E^{\prime} / E=0.78 /(5 \times\right.$ $\left.\left.10^{3}+\ell\right)\right]$, and - towards the origin $\left[E^{\prime} / E=-0.78 /\left(5 \times 10^{3}+\ell\right)\right]$. As can be seen from Eqs. (A.4)-(A.8), for $r>40 \mathrm{Mpc}$ the considered model becomes the homogeneous Friedmann model, which is in this particular case the $\Lambda$ CDM model. First light propagates towards the center, $E^{\prime}>0$, and after passing through the origin, $E^{\prime}$ becomes negative, and so on. Because the inhomogeneous blocks are matched to the Friedmann model the average density is almost the same as in the $\Lambda \mathrm{CDM}$ model

$$
\begin{aligned}
\langle\rho\rangle & =\frac{1}{V} \int_{0}^{r} \mathrm{~d} r \int_{-\infty}^{-\infty} \mathrm{d} p \int_{-\infty}^{-\infty} \mathrm{d} q \frac{R^{2}}{E^{2}} \frac{R^{\prime}-R E^{\prime} / E}{\sqrt{1-k}} \rho \\
& =\frac{4 \pi}{V} \int_{0}^{r} \mathrm{~d} r \frac{R^{2} R^{\prime}}{\sqrt{1-k}} \rho=\frac{1}{V} \int_{0}^{r} \mathrm{~d} r \frac{M^{\prime}}{\sqrt{1-k}} \approx \frac{M_{0}}{V} .
\end{aligned}
$$

The first equality is implied by the dipole not contributing to the average Bolejko (2009). The last approximation is exact when curvature vanishes, although in the considered model $k<10^{-5}$, so it is quite an accurate approximation. Finally, the junction conditions (matching the Szekeres to Friedmann model) imply that both the total mass and volume of the inhomogeneous patch are the same as in the homogeneous model. Thus, the average density of the Szekeres Swiss-Cheese model is almost the same 
as the background model - the $\Lambda \mathrm{CDM}$ model. This is also true for the Hubble parameter

$$
\begin{aligned}
\langle H\rangle & =\frac{1}{V} \int_{0}^{r} \mathrm{~d} r \int_{-\infty}^{-\infty} \mathrm{d} p \int_{-\infty}^{-\infty} \mathrm{d} q \frac{R^{2}}{E^{2}} \frac{\left(R^{\prime}-R E^{\prime} / E\right)^{2}}{\sqrt{1-k}} \\
& \times \frac{1}{3} \frac{\dot{R}^{\prime}+2 \dot{R} R^{\prime} / R-3 \dot{R} E^{\prime} / E}{R^{\prime}-R E^{\prime} / E} \\
& =\frac{4 \pi}{3 V} \int_{0}^{r} \mathrm{~d} r \frac{R^{2} R^{\prime}}{\sqrt{1-k}}\left(\frac{\dot{R}^{\prime}}{R^{\prime}}+2 \frac{\dot{R}}{R}\right) \\
& \approx \frac{4 \pi}{3 V} \int_{0}^{r} \mathrm{~d} r\left(R^{2} \dot{R}\right)^{\prime}=\frac{\dot{R}_{0}}{R_{0}}=H_{0} .
\end{aligned}
$$

The redshift is obtained for null geodesic equations. The axially null geodesic (propagation along the axial axis) is

$\frac{\mathrm{d} t}{\mathrm{~d} r}= \pm \frac{R^{\prime}-R E^{\prime} / E}{\sqrt{1-k}}$,

and the redshift relation is

$\ln (1+z)= \pm \frac{1}{c} \int_{r_{e}}^{r_{o}} \mathrm{~d} r \frac{\dot{R}^{\prime}-\dot{R} E^{\prime} / E}{\sqrt{1-k}}$

or equivalently

$$
\begin{aligned}
& \frac{\mathrm{d} r}{\mathrm{~d} z}= \pm \frac{1}{1+z} \frac{\sqrt{1-k}}{\dot{\dot{R}^{\prime}-\dot{R} E^{\prime} / E},} \\
& \frac{\mathrm{d} t}{\mathrm{~d} z}=\frac{1}{1+z} \frac{R^{\prime}-R E^{\prime} / E}{\dot{R}^{\prime}-\dot{R} E^{\prime} / E} .
\end{aligned}
$$

\section{References}

Alcaniz, J. S. 2002, PRD 65, 123514

Alnes, A., \& Amarzguioui, M. 2006, PRD, 74, 103520

Alnes, H., Amarzguioui, M., \& Grøn, Ø. 2006, PRD, 73, 083519

Barrow, J. D., \& Ottewill, A. C. 1983, JPA, 16, 2757

Barrow, J. D., \& Cotsakis, S. 1988, PLB, 214, 515

Bento, M., \& Bertolami, O., \& Sen, A. A. 2003, PLB, 575, 172

Bertolami, O., Sen, A. A., Sen, S., \& Silva, P. T. 2004, MNRAS, 53, 329

Bolejko, K. 2008, PMC Phys. A, 2, 1

Bolejko, K. 2009, GRG, 41, 1737

Bolejko, K., \& Célérier, M. N. 2010, PRD, 82, 103510

Bolejko, K., \& Wyithe, J. S. B. 2008, JCAP, 02, 020
Bolejko, K., \& Lasky, P. 2008, MNRAS, 391, L59

Bolejko, K., Krasiński, A., Hellaby, C., \& Célérier, M. N. 2009, Structures in the Universe by Exact Methods: Formation, Evolution, Interactions, (Cambridge: Cambridge University Press)

Bonvin, C., Durrer, R., \& Gasparini, M. A. 2006, PRD, 73, 023523

Buchdahl, H. A. 1970, MNRAS, 150, 1

Caldwell, R. R., Kamionkowski, M., \& Weinberg, N. N. 2003, PRL, 91, 071301

Célérier, M. N. 2000, A\&A, 353, 63

Célérier, M. N., Bolejko, K., \& Krasiński, A. 2010, A\&A, 518, A21

Chiba, T., Okabe, T., \& Yamaguchi, M. 2000, PRD, 62, 023511

Clarkson, C., Ananda, K., \& Larena, J. 2009, PRD, 80, 083525

Clifton, T., Ferreira, P. G., \& Land, K. 2008, PRL, 101, 131302

Copeland, E. J., Sami, M., \& Tsujikawa, S. 2006, IJMPD, 15, 1753

Debnath, U., Banerjee, A., \& Chakraborty, S. 2004, CQG, 21, 5609

Deffayet, C. 2001, PLB, 502, 199

Dyer, C. C., \& Roeder, R. C. 1972, ApJ, 174, L115

Dvali, G. R., Gabadadze, G., \& Porrati, M. 2000, PLB, 485, 208

Enqvist, K., \& Mattsson, T. 2007, JCAP, 02, 019

Ellis, G. F. R. 1971, Proceedings of the International School of Physics "Enrico Fermi", Course 47: General Relativity and Cosmology, ed. R. K. Sachs. reprinted, with historical comments: 2009, GRG, 41, 581, 104 (New York and London: Academic Press)

García-Bellido, J., \& Haugbølle, T. 2008, JCAP, 04, 003

Gurzadyan, V. G., \& Kocharyan, A. A. 2009, A\&A, 493, L61

Hoyle, F., \& Vogeley, M. S. 2004, ApJ, 607, 751

Hsu, S. D. H. 2004, PLB, 594, 13

Hui, L., \& Greene, P. B. 2006, PRD, 73, 123526

Iguchi, H., Nakamura, T., \& Nakao, K. 2002, PTP, 108, 809

Jones, D. H., Read, M. A., Saunders, W., et al. 2009, MNRAS, 399, 683

Kaloper, N., \& Linde, A. D. 1999, PRD, 60, 103509

Kerner, R. 1982, GRG, 14, 453

Koyama, K. 2008, GRG, 40, 421

Kristian, J., \& Sachs, R. K. 1966, ApJ, 143, 379

Li, B., \& Barrow, J. D. 2007, PRD, 75, 084010

Li, M. 2004, PLB, 603, 1

Maia, M. D., Monte, E. M., Maia, J. M. F., et al. 2005, CQG, 22, 1623

Moldenhauer, J., Ishak, M., Thompson, J., \& Easson, D. A. 2010, PRD, 81, 063514

Mukhanov, V. 2005, Physical Foundations of Cosmology (Cambridge: Cambridge University Press, Cambridge)

Peebles, P. J. E. 1980, The Large-Scale Structure of the Universe, (Princeton: Princeton University Press)

Peebles, P. J. E. 2010, AIP Conf. Proc., 1241, 175

Peebles, P. J. E., \& Ratra, B. 2003, RMP, 75, 559

Pyne, T., \& Birkinshaw, M. 2004, MNRAS, 348, 581

Randall, L., \& Sundrum, R. 1999, PRL, 83, 3370

Räsänen, S. 2009, JCAP, 02, 011

Sachs, R. K. 1961, Proc. Roy. Soc. London A, 264, 309

Sahni, V., \& Wang, L. 2000, PRD, 62, 103517

Sahni, V., \& Shtanov, Y. 2003, JCAP, 11, 014

Saini, T. D., Raychaudhury, S., Sahni, V., \& Starobinsky, A. A. 2000, PRL, 85, 1162

Simpson, F. 2007, JCAP, 03, 016

Starobinsky, A. A. 1980, PLB, 91, 99

Vanderveld, R. A., Flanagan, E. E., \& Wasserman, I. 2007, PRD, 76, 083504 